\title{
Actividad antimicótica de nanopartículas
}

\author{
Laura E. Castrillón Rivera, *Alejandro Palma Ramos **
}

RESUMEN: La terapia antifúngica tiene como principal desventaja que existen pocas moléculas que carecen de acción citotóxica hacia las células de mamífero, ya que comparten muchas características funcionales porque también son células eucarióticas. Por otra parte, debido a la naturaleza química de muchas de estas moléculas que son poco solubles en agua, este hecho puede afectar su biodisponibilidad. Lo anterior limita su acción terapéutica y exige el diseño de formas farmacéuticas efectivas. Por esta razón, el desarrollo de nuevas tecnologías y síntesis de nanomateriales surge como una posible alternativa para el tratamiento de las infecciones por hongos. En este trabajo de revisión se presentan los desarrollos nanotecnológicos con miras a la obtención de formas farmacéuticas eficientes y seguras de los antimicóticos como son los liposomas, nanopartículas de plata, nanopartículas lipídicas sólidas, nanopartículas poliméricas y microtúbulos lipídicos.

PALABRAS CLAVE: antimicóticos, nanopartículas, antifúngicos, actividad antimicótica

ABSTRACT: Antifungal therapy has as major disadvantage that there is little free molecules of cytotoxic action towards mammalian cells and which share many characteristics that are also functional eukaryotic cells. Moreover, due to the chemical nature of many of these molecules that are poorly soluble in water this can affect their bioavailability. This limits its therapeutic action and require the design of effective dosage forms. For this reason, the development of new technologies and synthesis of nanomaterials is emerging as a possible alternative for the treatment of fungal infections. Nanotechnological developments aimed at obtaining efficient and safe antifungal dosage forms such as liposomes, silver nanoparticles, solid lipid nanoparticles, polymeric and lipid microtubules nanoparticles are presented in this review paper.

KEYWORDS: antifungal, nanoparticles, antifungal, antifungal activity

\section{Importancia de su divulgación}

El aumento de la incidencia de patologías en las que los hongos aparecen como patógenos emergentes se ha asociado con la susceptibilidad en pacientes que presentan cierto grado de inmunodeficiencia. Estas infecciones se observan principalmente como hongos oportunistas. Los principales factores de riesgo para estas infecciones son la neutropenia, diabetes, cirugías,

* Doctora en biología clínica por la Escuela Nacional de Ciencias Biológicas IPN. Profesor investigador de la Universidad Autónoma Metropolitana Unidad Xochimilco (UAM-X). Departamento de Sistemas Biológicos.

Correspondencia: (lcrivera@correo.xoc.uam.mx).

** Maestro en ciencias en biomedicina por la Escuela Nacional de Ciencias Biológicas IPN. Profesor investigador UAM-X. Departamento de Sistemas Biológicos. 
infecciones nosocomiales y tratamientos excesivos con antibióticos o inmunosupresores para pacientes transplantados, entre otros. La aparición de nuevos gérmenes así como la aparición del fenómeno de resistencia hacia los antifúngicos y su elevada toxicidad ha favorecido el desarrollo de síntesis de nuevas moléculas con potencial antifúngico sin alcanzar aún los resultados deseados. Por esta razón, el desarrollo de nuevas tecnologías y síntesis de nanomateriales surge como una posible alternativa para el tratamiento de las infecciones por hongos. En este trabajo se presentan los avances logrados en este campo donde se ha demostrado que con el empleo de nanoformulaciones, un gran número de fármacos han mejorado su función terapéutica, reduciendo las dosis necesarias, así como las reacciones adversas.

El abuso de la terapia antibiótica, el uso de inmunosupresores como terapia contra el cáncer o para tratamientos postransplante así como cualquier condición que favorezca un estado de debilidad inmunitaria son algunas de las condiciones que favorecen la aparición de infecciones entre las que se encuentran las micosis (Castrillón y Palma, 2012).

Las micosis se clasifican de acuerdo con su localización anatómica como micosis cutáneas, micosis subcutáneas, micosis profundas y micosis oportunistas. Para su tratamiento se utilizan antifúngicos (antimicóticos) que son cualquier sustancia capaz de alterar las estructuras celulares del hongo que logre inhibir su desarrollo, alterando su viabilidad o capacidad de supervivencia.

La clasificación de los antimicóticos como se observa en la tabla 1 se realiza de acuerdo con su estructura química, origen (biológico/sintético), mecanismo de acción (fungistático/fungicida), vía de administración y toxicidad entre otros (Allevato et al., 2007).

Aunque actualmente existe una variedad limitada de antifúngicos, sus efectos adversos, fracasos terapéuticos y la aparición de resistencia son problemas importantes que aparecen con el empleo de estos fármacos. Además, otras de las limitaciones para el uso de estas sustancias son su baja solubilidad en solventes acuosos lo que afecta directamente su biodisponibilidad, su elevada toxicidad por acción citotóxica hacia tejidos sanos, su rápida degradación y aclaramiento del torrente circulatorio, muchos de ellos de difícil acceso a través de las membranas celulares por lo que su actividad hacia microrganismos es baja y en consecuencia su efecto antibiótico también. Por esta razón, se requiere de dosis altas para alcanzar su efecto terapéutico y, por tanto, aumentar el riesgo de los posibles efectos adversos relacionados con estas sustancias.

Estos problemas pueden ser evitados, en parte, utilizando asociaciones de antifúngicos, desarrollando nuevas moléculas, o bien encontrando sistemas de entrega de fármacos alternativos como son las nanopartículas (NPs) con fármacos a través de encapsulación física, adsorción o conjugación 


\begin{tabular}{|c|c|c|c|c|c|c|}
\hline 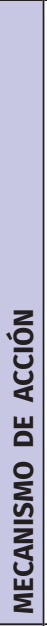 & 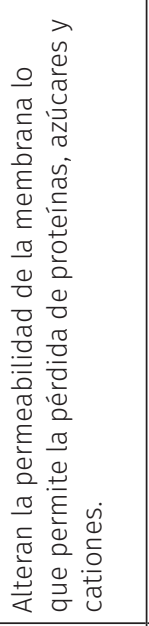 & 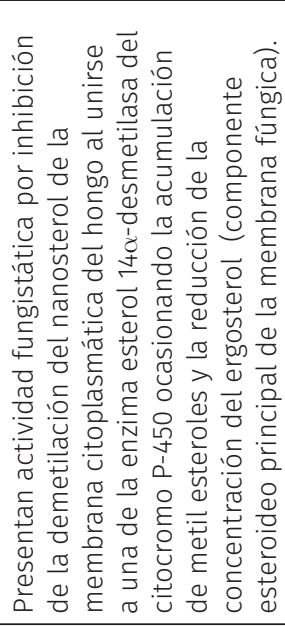 & 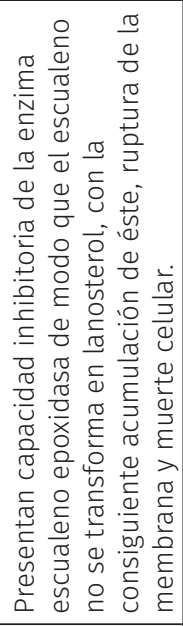 & 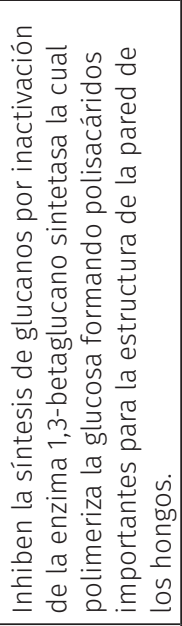 & 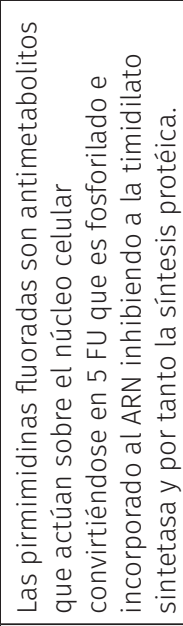 & 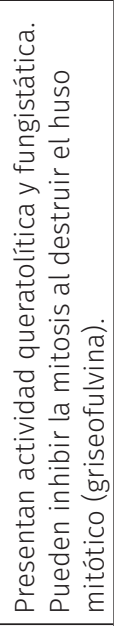 \\
\hline 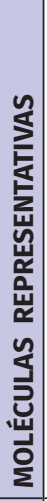 & 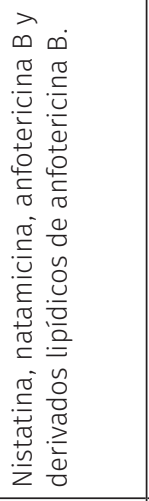 & 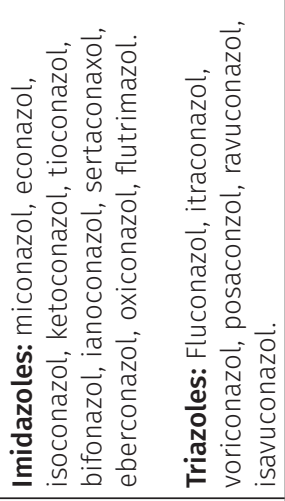 & 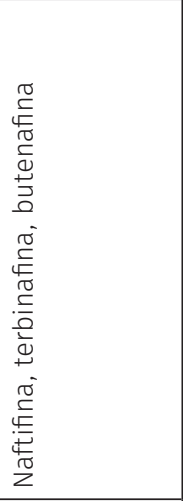 & 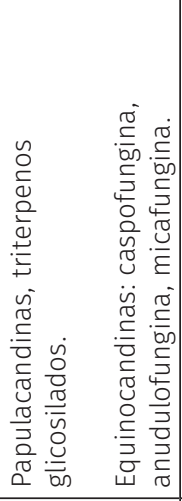 & 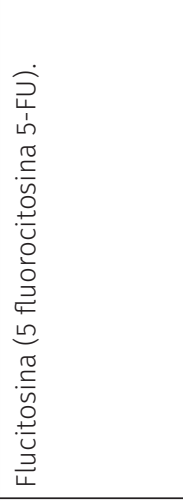 & 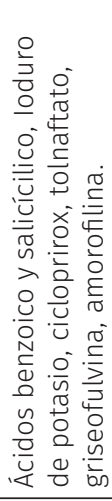 \\
\hline 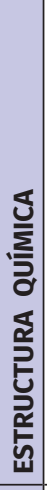 & 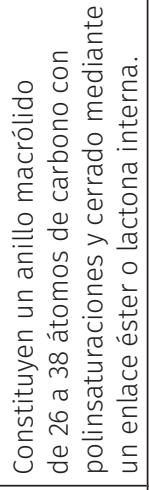 & 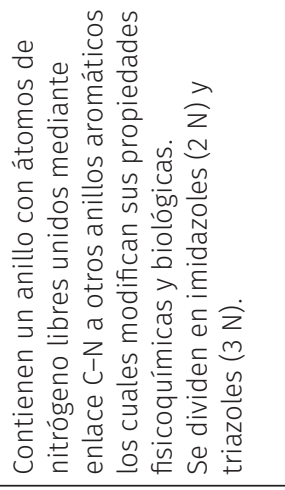 & 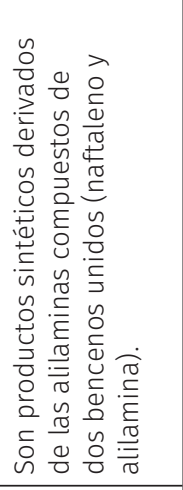 & 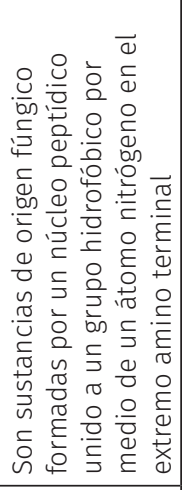 & 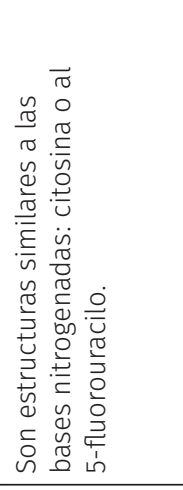 & 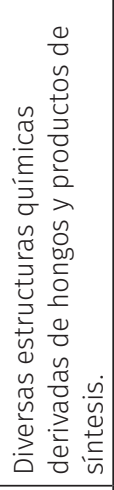 \\
\hline 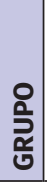 & 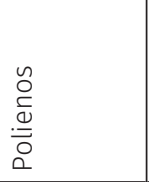 & $\begin{array}{l}\cong \\
\frac{\Phi}{O} \\
\frac{N}{<}\end{array}$ & 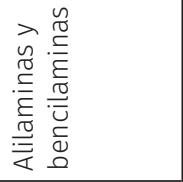 & 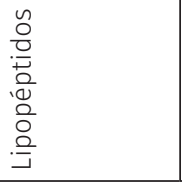 & 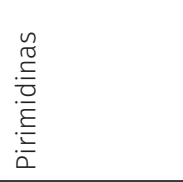 & \\
\hline
\end{tabular}


química, pues esta presentación modifica los índices farmacocinéticos y terapéuticos de estas sustancias y pueden mejorar significativamente en contraste con el fármaco libre. Se han reconocido muchas ventajas en estos sistemas incluyendo mejoras en la solubilidad, prolongar el tiempo de vida en circulación sistémica, liberación controlada y la entrega en tejidos y células de interés y la entrega de múltiples agentes terapéuticos como terapia combinada (Zhang et al., 2010).

Los materiales nanoestructurados tienen propiedades fisicoquímicas únicas como su tamaño ultrapequeño (1-100 nm) y controlable, gran área de superficie, alta reactividad, interacciones biológicas únicas y estructuras funcionalizables. Estas propiedades facilitan la administración de drogas antimicrobianas que rebasan algunas de las limitaciones en la terapéutica antimicrobiana. A continuación se presentan los principales avances en el desarrollo y eficacia de estos materiales relacionados con fármacos de acción antifúngica.

\section{Liposomas}

Los liposomas son unas pequeñas vesículas huecas cuya membrana doble está compuesta de una doble capa de fosfolípidos. Estos fosfolípidos constan de una cabeza hidrosoluble en forma de esfera y de una cola liposoluble. Los fosfolípidos naturales se obtienen de la soya, y en una solución acuosa forman la estructura esférica conocida como liposoma. Durante este proceso las colas lipófilas de los fosfolípidos entran en contacto entre ellas formando una membrana de doble capa que es hidrófila en sus partes exteriores y lipófila en su interior. Por lo tanto, el interior y el exterior de un liposoma es hidrosoluble y el interior de la membrana del liposoma es liposoluble (Kant et al., 2012; Tochilin, 2005).

Los liposomas multilaminares constan de liposomas unilaminares de distintos tamaños que están entrelazados: un liposoma pequeño encerrado en otro mayor y estos dos, a su vez, encapsulados dentro de otro todavía mayor, y así sucesivamente. Como resultado se obtiene un liposoma multilaminar formado por hasta 12 liposomas de diferentes tamaños, siendo lo habitual utilizar de 5 a 7 encapsulados. El tamaño de estos liposomas entrelazados varía entre 20 y 300 nanometros. Existe una relación directa entre el nivel de penetración de los liposomas y su tamaño, penetrando más cuanto menor el tamaño (Jesorka y Orwar, 2008).

La actividad antimicrobiana de estas estructuras se debe a que los los liposomas se fusionan con las membranas de los microrganismos y entregan su carga al interior de las células. El polietilen glicol (PEG) se ha utilizado frecuentemente en la superficie del liposoma para crear un escudo protector que prolonga el tiempo de vida media de estas estructuras en la circulación sanguínea, formando una capa de hidratación que retrasa el reconocimiento y captura por el sistema retículo endotelial (Immordino et al., 2006). 
Por otra parte, la unión de ligandos específicos como segmentos de anticuerpos, aptameros, péptidos y pequeñas moléculas en la superficie del liposoma puede unir selectivamente a los microrganismos o a células infectadas y liberar al fármaco para matar o inhibir el crecimiento de éstos.

La primera nanomedicina puesta en el mercado para administración intravenosa en el tratamiento de infecciones fúngicas severas fue Ambisome ${ }^{\circledR}$, en el año de 1991, corresponde a la formulación de anfotericina B liposomal unilaminar de fosfolípidos y coleserol de tamaño $80 \mathrm{~nm}$ la cual demostró su actividad en el año 1981 sobre Leishmania que es un parásito intracelular y para el año de 1982 se reportó su eficacia sobre los hongos Cryptococcus e Histoplasma (Grabill et al., 1982; Taylor et al., 1982), esta presentación permitió reducir entre 50 y 70 veces la toxicidad del antifúngico observada con la formulación tradicional (asociación desoxicolato y antifúngico) por lo que su administración en pacientes se ve aumentada 5 veces. El éxito de esta formulación se relacionó con la capacidad de los liposomas por alcanzar y concentrarse en las células del sistema monocito-macrofágico.

AmBisome $\subseteq$ ha sido aprobada por la FDA y se ha utilizado en el tratamiento contra Candida spp, Aspergillus spp, Fusarium spp, en pacientes neutropénicos y en leishmaniasis visceral. La anfotericina B (AMB) (figura 1) se intercala en la bicapa lipídica de fosfolípidos en la membrana del liposoma. La entrega de AMB ocurre por unión a la pared celular del hongo y a través de mecanismos de absorción y disgregación de la membrana celular.

El marcaje fluorescente de AmBisome en ratones infectados con Candida ha permitido la localización de estos liposomas en los sitios infectados por este hongo.

Las formulaciones comerciales de AmB son AmBisome(c) son muy estables en estado de gel a temperatura ambiente con un tiempo de vida media en sangre prolongado y buena penetración en los tejidos. La mayor parte de los estudios de las formulaciones disponibles se han realizado con esta presentación (Barrrat y Bretagne, 2007).

El mayor problema de AmBisome $\subset$ sigue siendo su costo (estimado en 2004 en unos 16,000-20,000 euros/mes, frente a 127 euros para la formulación convencional); sin embargo, se puede justificar debido a la disminución de los gastos derivados de los efectos adversos asociados al uso de la presentación convencional de anfotericina B (Irache, 2010).

FIGURA 1. Estructura química de la anfotericina B.<smiles>CC(C)C(C)/C=C/C=C/C=C/C=C/C=C/C=C/C=C/C(CC(O)C(C)C(O)CC(=O)CC(O)CC(O)C(O)CCC(O)CC(O)CC(=O)OC(C)C(C)C(C)O)OC1OC(C)C(O)C(N)C1O</smiles> 
Por otra parte, la toxicidad aguda que ocasiona la nistatina clásica por vía intravenosa (i.v.) impide su uso para micosis invasoras por lo que se ha desarrollado una presentación liposomal multilaminar de este antifúngico la cual ha demostrado buena actividad y baja toxicidad, actualmente se encuentra en ensayos clínicos en fase II en los Estados Unidos y en Europa (Carrillo-Muñoz et al., 1999).

\section{Nanopartículas de plata (NP-Ag)}

La plata tiene propiedades médicas conocidas por más de 2 mil años y se ha utilizado como agente antimicrobiano. La plata se utiliza generalmente en su forma de nitrato para inducir su efecto antimicrobiano. Las NP de plata (NP-Ag o nanoplata son nanopartículas de un rango de 1 a $100 \mathrm{~nm}$ de tamaño, se utilizan por el gran aumento de su área superficial que se expone hacia los microrganismos, por lo cual sus principales aplicaciones son el tratamiento de heridas, materiales dentales, recubrimiento de materiales de acero inoxidable, textiles, tratamiento de aguas, protectores solares, ya que estas partículas han demostrado baja toxicidad hacia las células humanas, elevada estabilidad térmica y baja volatilidad (Juneyoung et al., 2010).

El mecanismo de acción antifúngica de estas NPs se debe a que estas partículas se unen a la membrana del hongo modificando su permeabilidad y con ello alterar la viabilidad celular, o bien, pueden penetrar y modificar la capacidad respiratoria, detener su división celular ocasionando así la muerte celular. También se ha reportado que las NP-Ag pueden liberar iones de plata que interactúan con los grupos tiol de muchas enzimas inactivándolas o bien generar radicales libres que son extremadamente reactivos y presentan gran actividad citotóxica (Prabhu y Poulose, 2012).

Los reportes de actividad contra Candida albicans mencionan que estas NP-Ag inhiben eficientemente el crecimiento de levaduras a concentraciones por debajo del límite citotóxico probado en fibroblastos humanos a concentraciones de $30 \mathrm{mg} / \mathrm{mL}$ de plata en contraste con la plata iónica que inhibe el crecimiento a concentraciones de $1 \mathrm{mg} / \mathrm{L}$ en este mismo modelo experimental (Panácek et al., 2009); además, se ha documentado la capacidad inhibitoria de la gemación de esta levadura por la dispersión de la integridad de su membrana por acción de estas nanopartículas (Kim et al., 2009).

En relación con la actividad antifúngica comparativa de las NP-Ag con anfotericina B y fluconazol ante $C$. albicans y $S$. cerevisiae se ha demostrado que estas partículas muestran una actividad antifúngica potente con valores similares en las concentraciones mínimas inhibitorias (CMI) comparando con los antibióticos control (Nasrollahi et al., 2011). Otro efecto descrito para las NP-Ag se relaciona con su acción sobre el micelio en Candida ya que la presencia de suero induce la filamentación la cual se inhibe significativamente en presencia de estas nanopartículas (figura 2). 
FIGURA 2. Levaduras de Candida albicans (foto izquierda); filamentación de Candida albicans (foto derecha).

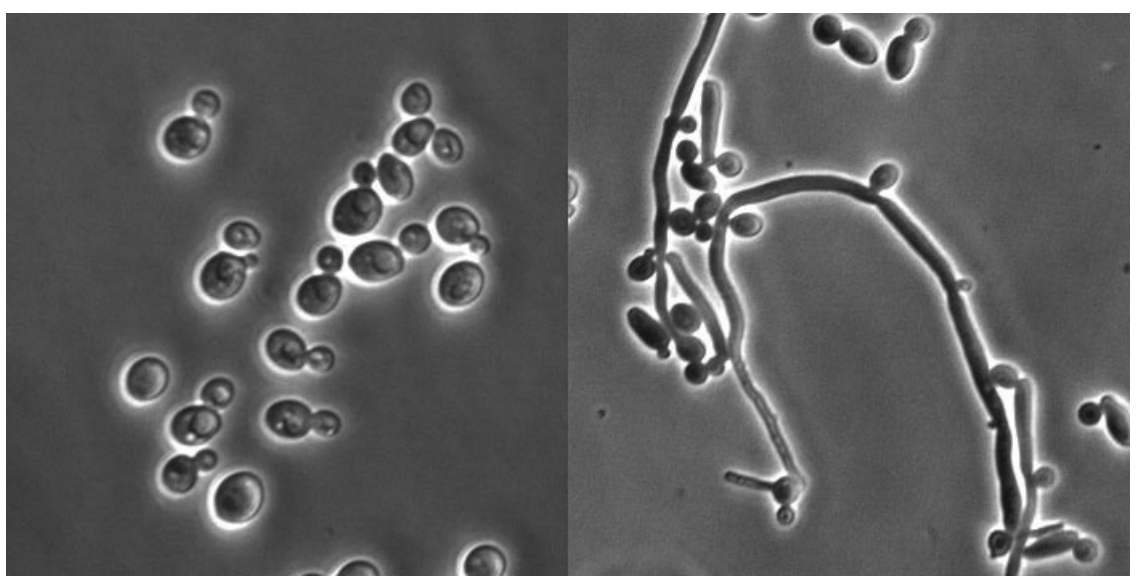

Fuente: <http://www.infectologiapediatrica.com/blog/tag/candida-albicans>.

En la búsqueda de respuestas antifúngicas por estas NPs, también se ha probado la acción citotóxica de estas nanopartículas sobre los dermatofitos demostrándose su actividad en aislamientos clínicos y en cepas de referencia de Trichophyton mentagrophytes con respuestas comprables con anfotericina B y respuestas aún mayores cuando se comparan contra el fluconazol (KeukJun et al., 2008).

Se han explorado otras acciones de estas nanoestructuras sobre hongos fitopatógenos como Bipolaris sorokiniana y Magnaporthe grisea demostrando que tanto las NP-Ag como los iones de plata interfieren sobre la formación de esporas y el desarrollo de la enfermedad promoviendo el contacto directo de la plata con las esporas y los tubos germinativos interfiriendo con su viabilidad (Young-Ki y Byung, 2009).

Resulta interesante que las nanopartículas de plata pueden ser sintetizadas biológicamente por bacterias, hongos y plantas, para el caso de los hongos éstos atrapan los iones de plata $\left(\mathrm{Ag}^{++}\right)$en la superficie celular y por las enzimas que ellos secretan reducen a estos iones. Aunque no se conoce con exactitud este mecanismo, la velocidad de este proceso es lenta por lo que actualmente se utilizan los extractos de plantas como mejores alternativas para la síntesis de estas nanopartículas, pues lo realizan en periodos de tiempo cortos (Savithramma et al., 2011).

Aunque las NP-Ag muestan actividad antimicrobiana considerable, también existen reportes adversos de estas estructuras sobre el ser humano y otros seres vivos así como al medio ambiente por su capacidad de liberar iones plata en fase acuosa. La plata es un ácido débil y su tendencia es a reaccionar con bases. La mayoría de las células contienen azufre y fósforo que son bases débiles. El ácido desoxirribonucléico o ADN contiene estos átomos 
por lo que estas nanopartículas pueden reaccionar con ellos, ocasionar su destrucción y, por lo tanto, llevar a la muerte celular.

Entre los efectos nocivos de las partículas de plata presentados en el humano son la argiria producida cuando las partículas de plata relativamente grandes quedan atrapadas debajo de la piel y luego reaccionan con la luz solar, convirtiendo la piel gris o azul, también puede presentarse la argirosis que se describe como una decoloración gris-azulada en las estructuras oculares como consecuencia de la acumulación de plata en la córnea (SánchezHuerta et al., 2006). Del mismo modo, se han descrito efectos hepatotóxicos por alteración del estrés oxidativo mitocondrial alterando la permeabilidad de iones potasio y sodio, así como hiperplasia de ductos biliares, necrosis, fibrosis y pigmentación. Otras afectaciones descritas son las células de riñón, piel, sangre y tractos respiratorio e intestinal en repuesta a las NP-Ag (Prabhu y Poulose, 2012).

El deterioro ambiental de estas partículas se relaciona con la incapacidad de discriminación entre los diferentes microrganismos presentes en el medio, destruyendo aquellos que son benéficos a la ecología. Otra acción de las nanopartículas de plata recae sobre las bacterias desnitrificantes alterando el proceso de desnitrificación que involucra la conversión de nitratos en gas nitrógeno, esencial para las plantas por lo que la pérdida de denitrificación a través de la reducción de la productividad de las plantas puede llevar a la eutroficación de ríos, lagos y destruir ecosistemas marinos (ibid.).

\section{Nanopartículas lipídicas sólidas (SLN y NLC)}

Las nanopartículas sólidas (SNLs) son una forma alternativa en la administración de fármacos lipofílicos, son sistemas particulados coloidales con una proporción de 70-95\% de agua, con diámetros de 50 a 1000 nm compuestos de lípidos en fase sólida a temperatura ambiente y surfactantes para emulsificación, son elaborados a partir de lípidos fisiológicos que tienden a gelificar y a expulsar el fármaco durante el almacenamiento, tienen capacidad de carga limitada, son biodegradables y con buena tolerancia. La adición de lípidos líquidos en los que el fármaco es más soluble genera nuevos sistemas nanoparticulares conocidos como acarreadores lipídicos nanoestructurados (NLC) (Garzón et al., 2008).

Se utilizan preferentemente para fármacos poco solubles en agua y son utilizados preferentemente como productos del cuidado de la piel ya que tienen propiedades oclusivas que permiten formar una capa delgada que reduce la evaporación del agua y retiene la humedad en la piel y prolonga la residencia del fármaco en el estrato córneo, por esta razón se promueve la penetración de moléculas en este tejido. Estos preparados son estables en agua y su facilidad para prepararlos hace posible su producción a gran escala y de manera reproducible. 
FIGURA 3. Estructura química del ketoconazol.

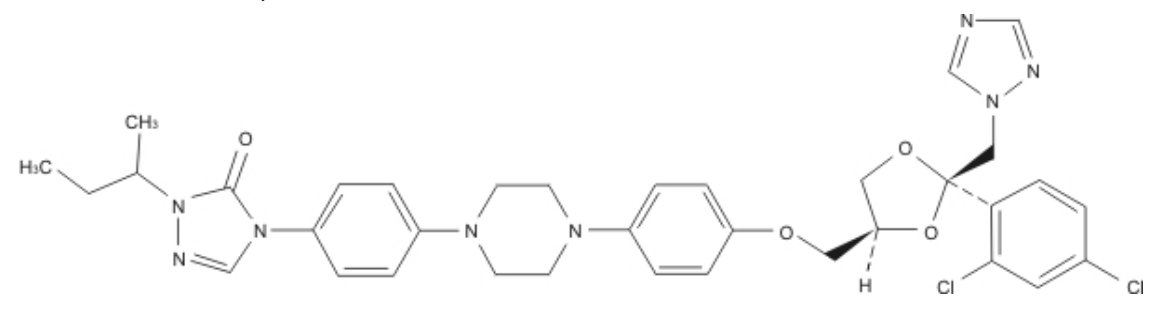

Los desarrollos nanotecnológicos relacionados con antimicóticos se han reportado principalmente para los azoles y su posible uso en aplicación tópica para las infecciones superficiales por hongos, entre los que se encuentran el ketoconazol clotrimazol, miconazol, econazol, oxiconazol y tioconazol, extremadamente insolubles en agua y susceptibles para la degradación por oxidación e hidrólisis por agentes químicos, lo cual limita su biodisponibilidad y, por tanto, sus efectos antifúngicos (Paolicelli et al., 2011; Souto et al., 2004; Souto y Müller, 2000; Sanap y Mohanta, 2013).

\section{Nanopartículas poliméricas}

Una de las propuestas tecnológicas más prometedoras para mejorar el tratamiento antifúngico son los sistemas de liberación y vectores farmacéuticos entre los que destacan las nanopartículas (NPs) poliméricas, éstas son partículas sólidas, biodegradables y coloidales con tamaños de 10 a $1000 \mathrm{~nm}$ e incluyen a las nanoesferas y nanocápsulas cuya diferencia reside en si el fármaco es absorbido en la superficie de la esfera o se encuentra encapsulado. Estas nanopartículas poliméricas son muy estables, se adaptan fácilmente a los procesos industriales de fabricación y se puede controlar su tamaño por síntesis modificando sus propiedades seleccionando la longitud de su polímero, surfactante y solvente orgánico y modificar su superficie para modular sus propiedades.

Existen dos tipos de nanopartículas poliméricas, una está formada por el autoensambe espontáneo de copolímeros que contienen segmentos hidrofílicos e hidrofóbicos, estos últimos forman un núcleo que incorpora los fármacos hidrofóbicos mientras que los segmentos hidrófilos protegen este núcleo de la opsonización y degradación. El otro tipo consiste en polímeros lineales de polialquilacrilatos y polimetilmetacrilato que forman nanocápsulas a través de un método de polimerización de emulsiones.

Las nanoesferas cargadas con anfotericina $B$ recubiertas con surfactante poloxamero 188 han demostrado baja eficacia terapéutica contra C. albicans comparada con la droga libre debido a la toxicidad presente por acumulación en hígado y riñón en animales neutropénicos.

Los azoles (econazol y clotrimazol) aumentan su biodisponibilidad cuando se encapsulan en nanopartìculas de un polímero sintético (polilac- 
tide-co-glicolído o PLG) o en un polímero natural (alginato estabilizado con quitosán), estas formulaciones fueron probadas en ratón y los antifúngicos fueron analizados en plasma por cromatografía de líquidos de alta resolución. Estos fármacos se detectaron en pulmones, hígado y bazo hasta $8 \mathrm{~d}$ cuando se presentaron en forma encapsulada, en cambio la droga libre fue eliminada en 12 horas, por tanto la biodisponibilidad oral fue mejorada con el uso de estas partículas (Pandey et al., 2005).

En otro trabajo se evaluó la capacidad del econazol encapsulado en nanopartículas de alginato para reducir la carga hasta $90 \%$ en pulmones y bazo de ratones infectados con la bacteria $M$. tuberculosis, y debido a que su biodisponibilidad es pobre por vía oral, el econazol puede ser admninistrado a dosis alta y frecuente por dos veces por día cuando se ofrece con esta presentación (Ahmad et al., 2007).

Por otra parte, se ha demostrado la capacidad antifúngica contra T. rubrum de nanocápsulas y nanoemulsiones con el aceite esencial de Melaleuca alternifolia en modelo de infección en uña en donde las nanocápsulas mostraron mejor efecto (Flores et al., 2013).

\section{Microtubos lipídicos}

Los microtubos lipídicos consisten en bicapas empacadas concentricamente que se autoensamblan de un lípido diacetilenico, glicolípidos y fosfolípidos, forman cilindros de menos de $1 \mathrm{~mm}$ de diámetro durante un proceso de enfriamiento controlado. Las interacciones quirales entre las moléculas de lípidos causan que las bicapas giren y formen una estructura tubular. Estas estructuras sirven para la liberación sostenida de agentes bioactivos, se cargan con agentes bajo condiciones acuosas, son embebidas en hidrogeles de agarosa, son biocompatibles, seguros, no inducen procesos inflamatorios y son capaces de proporcionar la entrega hacia sitios específicos para proteínas, péptidos y ácidos nucléicos. A la fecha se han diseñado microtubos con proteínas como el factor transformante- $\beta$, factor de crecimiento neural y proteínas de alto peso molecular como cininógeno (Rawat et al., 2008).

Esta nanoestructura se ha utilizado para la incorporación de anfotericina B en microtubos formados de ácido 12-hidroxiesteárico (12HSA) cuando se mezcla con etanolamina en medio acuoso, su actividad antifúngica demostró resultados similares al preparado comercial de AmB (Fungizoneâ) que son micelas formadas con el detergente deoxicolato de sodio utilizadas en forma sistémica administrado por una perfusión lenta diluida al $5 \%$ con glucosa (Salerno et al., 2013).

\section{Conclusión}

En años recientes, la encapsulación de drogas antimicrobianas con actividad antifúngica en sistemas de nanopartículas ha emergido como una alternativa 
promisoria que aumenta la eficacia terapéutica y minimiza los efectos indeseables de estas drogas.

Las NP pueden imitar o modificar procesos biológicos y brindar soluciones a viejos problemas asociados con la solubilidad, biodisponibilidad, inmunocompatibilidad y citotoxicidad de muchos medicamentos de uso tradicional. Sin embargo, a pesar del progreso en la nanotecnología aplicada a los antifúngicos se ha observado como limitación para su uso la liberación prematura del fármaco de estas nanopartículas o la falta de la capacidad de blancos específicos para la entrega de estos antimicrobianos, por lo que se requiere la construcción de nanoestructuras con ligandos específicos que direccionen la captura para lograr mayor eficacia en la actividad de estas sustancias (Oropeza et al, 2012).

\section{Bibliografia}

Ahmad, Z., Sharma, S., Khuller, K. G. (2007) Chemotherapeutic evaluation of alginate nanoparticle-encapsulated azole antifungal and anitubercular drugs against a murine tuberculosis. Nanomedicine: Nanotechnology, Biology and Medicine, vol. 3: 239.

Allevato, J. M. A., Negroni, R., Galimberti, R. (2007) Antifúngicos. Ayer, hoy y mañana. Acta Terapéutica Dermatológica, vol. 30: 8.

Barratt, G., Bretagne, S. (2007) Optimizing efficcy of amphotericin B through nanomodification. Interntational Journal of Nanomedicine, vol. 2: 301.

Carrillo-Muñoz, A. J., Quindós, G., Tur, C. (1999) In vitro antifungal activity of liposomal nystatin in comparison with nystatin, amphotericin B cholesteryl sulphate, liposomal amphotericin B, amphotericin B lipid complex, amphotericin B desoxycholate, fluconazole and itraconazole. Journal of Antimicrobial and Chemotherapy, vol. 44: 397-401.

Castrillón, R. L., Palma, R. A. (2012) Inmunología de las micosis, cap. 10. En Méndez Tovar, L.J., López Martínez, R., Hernández Hernández, F. (comps.). Actualidades en micología médica. 6 ed. México: Ed. Sefirot-Ed. UNAM.

Garzón, L., Hernández, A., Vázquez, M. L., Villafuerte, L., García, B. (2008) Preparación de nanopartículas sólidas lipídicas (SLN) y de acarreadores lipídicos nanoestructurados (NLC). Revista Mexicana de Ciencias Farmacéuticas, vol. 39: 50.

Graybill, J. R., Craven, P. C., Taylor R. L. (1982) Treatment of murine Cryptococcus with liposome-associated amphotericin B. Journal of Infection Disease, vol. 145: 748.

Immordino, M. L., Dosio, F., Cattel, L. (2006). Stealth liposomes: Review of the basic science, rationale and clinical applications, existing and potential. International Journal of Nanomedicine, vol. 1: 297.

Jesorka, A., Orwar, O. (2008) Liposomes: Technologies and analytical applications. Annual Review of Analytical Chemistry, vol. 1: 801.

Juneyoung, L., Kim, K. J., Sung, S. W., Kim H. J., Lee, G. D. (2010) The silver nanoparticle (Nano-Ag): A new model for antifungal agents, chap. 15. En 
Nanotechnology and Nanomaterials Silver nanoparticles, Intechopen, marzo: pp. 295-308.

Kandile, G. N., Zaky, T. H., Mohamed, I. M., Mohamed, M. H. (2010) Silver nanoparticules effect on antimicrobial and antifungal activity of new heterocycles. Bulletin Korean Chemical Society, vol. 31: 12.

Kant, S., Kumar, S., Prashar, B. (2012) A complete review on: Liposomes. International Research Journal of Pharmacy, vol. 3: 10.

Keuk-Jun, K., Sung, S.W., Moon, S. K., Choi, J.S., Kim G. J. (2008) Antifungal effect of silver nanoparticles on dermatophytes. Journal Microbiology and Biotechnology, vol. 18: 1482.

Kim, K.J., Sung, W. S., Suh, B. K., Choi, J.S., Kim, J.G., Lee, D.G. (2009) Antifungal activity and mode of action of silver nano-particles on Candida albicans. Iometals, vol. 22: 235.

Nasrollahi, A., Pourshamsian, Kh., Manosourkiaee, P. (2011) Antifungal activity of silver nanoparticles of silver nanoparticules on some fungi. International Journal of Nano Dimension, vol. 1: 233.

Oropeza-Nuñez, R.M., Jáuregui-Haza, J.U. (2012) Las nanopartículas como portadores de fármacos: características y perspectivas. Revista CENIC Ciencias Biológicas, vol. 43: sep.-dic.

Panácek, A., Kolár, M., Vecerová, R., Prucek, R., Soukupová, J., Krystof, V., Hamal, P., Zboril, R., Kvítek, L. (2009) Antifungal activity of silver nanoparticles against Candida spp. Iomaterials, vol. 30: 6333.

Pandey, R., Ahmand, Z., Sharma, S., Khuller, G.K. (2005) Nano-encapsulation of azole antifungals: Potential applications to improve oral drug delivery. International Journal of Pharmaceutics, vol. 301: 268.

Paolicelli, P., Corrente, F., Serricchio, D., Cerreto, F., Cesa, S., Tita, B., Vitali, F., D'Auria, F.D., Simonetti, G., Casadei, MA. (2011) The system SLN-Dextran hydrogel: An application for the topical delivery of ketoconazole. Journal of Chemical and Pharmaceutical Research, vol. 3: 410.

Prabhu, S., Poulose, K.E. (2012) Silver nanoparticles: Mechanism of antimicrobial action, synthesis, medical applications, and toxicity effects. International Nano Letters, vol. 2: 32.

Rawat, M., Singh, D., Saraf, S., Saraf, S. (2008) Lipid carriers: A versatile delivery vehicle for protein and peptides. Yakugaku Zasshi, vol. 128: 269.

Ribeiro, S.A., Kiill, P., Kolenyak, F., Marielli da Luz, G., Rocha, H., Marlus, Ch., Palmira, D.G. (2012) Nanotechnology-based drug delivery system for dermatomycosis treatment. Current Nanoscience, vol. 8: 512.

Salerno, C., Chiappetta, A.D., Arechavala, A., Gorzalczany, S. (2013) Lipid-based microtubes for topical delivery of Amphotericin B. Colloids \& Surfaces B: Biointerfaces. vol. 107: 160.

Sanap, G.S., Mohanta, G.P. (2013) Design and evaluation of miconazole nitrate loaded nanostrucutred lipid carriers (NLC) for improving the antifungal therapy. Journal of Applied Pharmaceutical Science, vol. 3: 46.

Sánchez-Huerta, V., De-Witt, C.G., Hernández-Quintela, E., Rodríguez, A.A., 
Naranjo-Tackman, R. Argirosis corneal. (2006) Revista Mexicana de Oftalmología, vol. 80: 150 .

Savithramma, N., Linga, R.M., Rukmini, K., Suvarnalatha, D.P. (2011) Antimicrobial activity of silver nanoparticles synthesized by using medicinal plants. International Journal of Chem Tech Research, vol. 3: 1394.

Souto, E.B., Müller, R.H. (2005) SLN and NLC for topical delivery of ketoconazole. Journal of Microencapsulation, vol. 22: 501.

Souto, E.B., Wissing, S.A., Barbosa, C.M., Müller, R.H. (2004) Development of a controlled release formulation based on SLN and NLC for topical clortrimazole delivery. International Journal of Pharmaceutics, vol. 278: 71.

Taylor, R.L., Williams, D.M., Craven, P.C. (1982) Amphotericin B in liposomes: a novel therapy for histoplasmosis. The American Review of Respiration Disease, vol. 125: 610 .

Tochilin, P.V. (2005) Recent advances with liposomes as pharmaceutical carriers. Nature Reviews Drug Discovery, vol. 4: 145.

Yang, W., Widerhold, N.P., Williams, R.O.(2008) Drug delivery strategies for improved azole antifungal action. Expert Opinion Drug Delivery, vol. 5: 1199.

Young-Ki, J., Byung, H.K. (2009) Antifungal activity of silver ions and nanoparticles on phytopathogenic fungi. Plant Disease, vol. 93: 1037.

Zhang, L., Prornpattananangkul, D., Hu, C.M.J., Huang., C.M. (2010) Development of nanoparticles for antimicrobial drug delivery. Current Medicinal Chemistry, vol. 17: 585 . 